\title{
Endotelial activation and flow-mediated vasodilation in young patients with breast cancer
}

\author{
P. TESAROVA ${ }^{1, *}$, M. KALOUSOVA ${ }^{2}$, T. ZIMA ${ }^{2}$, M. SUCHANEK ${ }^{3}$, I. MALIKOVA ${ }^{4}$, J. KVASNICKA ${ }^{4}$, D. DUSKOVA ${ }^{5}$, V. TESAR $^{6}$, J. VACHEK $^{6}$, \\ Z. KRUPICKOVA-KASALOVA7 , J. MALIK ${ }^{7}$
}

\begin{abstract}
${ }^{1}$ Department of Oncology, 1st Faculty of Medicine, Charles University and General University Hospital, Prague, Czech Republic; ${ }^{2}$ Institute of Clinical Biochemistry and Laboratory Diagnostics, 1st Faculty of Medicine, Charles University and General University Hospital, Prague, Czech Republic; ${ }^{3}$ Institute of Chemical Technology, Prague, Czech Republic; ${ }^{4}$ Department of Clinical Haematology and Thrombotic Centre, General University Hospital, Prague, Czech Republic; ${ }^{5}$ Department of Blood Transfusion, General University Hospital, Prague, Czech Republic; ${ }^{6}$ Department of Nephrology, 1st Faculty of Medicine, Charles University and General University Hospital, Prague, Czech Republic; ${ }^{7} 3 \mathrm{rd}$ Department of Internal Medicine, 1st Faculty of Medicine, Charles University and General University Hospital, Prague, Czech Republic
\end{abstract}

*Correspondence: tesarova.petra@seznam.cz

Received February 3, 2013 / Accepted April 10, 2013

\begin{abstract}
Endothelial activation and dysfunction may play a significant role in the progression of breast cancer. In our study we examined markers of endothelial activation (soluble ICAM-1, P-selectin, E-selectin) in 98 young patients with breast cancer ( $<40$ years). In 50 of them (and 20 age-matched controls) we also measured flow mediated vasodilation. Patients with breast cancer had significantly higher serum levels of soluble E-selectin, P-selectin and ICAM-1, P-selectin was higher in patients with larger tumors, node involvement and seemed to be a predictor of poor outcome. We were unable to find significant difference in the parameters of flow mediated vasodilation between patients with breast cancer and healthy subjects, although both peak blood flow (PBF) and flow mediated vasodilation (FMD) seemed to be skewed compared to healthy subjects toward mean and lower levels. Cluster analysis enabled us to distinguish several larger groups of patients with different degree of endothelial activation and function and different outcome. Group of patients with high E-selectin, high ICAM-1 (higher endothelial activation) and low VEGF (putative endothelial damage) had more frequently negative estrogen receptors and had worse outcome compared to the group of patients with lower E-selectin, lower ICAM-1 and mostly positive estrogen receptors. Further studies of larger groups of patients should help to identify the pannel of endothelial markers which could help in predicting the outcome of young patients with breast cancer.
\end{abstract}

Key words: breast cancer, endothelial dysfunction, flow mediated vasodilation, adhesion molecules

One of the most important functions of vascular endothelium is the regulation of the permeability of vascular wall for cells and macromolecules. Endothelium is also able to regulate the regional blood perfusion by the local production of both vasodilatory (e.g. nitric oxide, prostacycline and endothelium hyperpolarizing factor) and vasoconstrictory factors (e.g. endothelin-1). Extravasation of leukocytes is mediated by the increased expression of different adhesion molecules (E-selectin, P-selectin, ICAM-1, VCAM-1, PECAM-1) by endothelial cells.

Endothelial damage usually results in prevailing vasoconstriction of the affected vessel and, if the integrity of the endothelial barrier is perturbed, activation of von Willebrand factor with subsequent stimulation of platelet aggregation and activation of the coagulation cascade may ensue. In the same time endothelium may also counteract the coagulation by the activation of anticoagulation (binds antithrombin III and produces thrombomodulin) and fibrinolysis (by synthetising both tissue plasminogen activator - tPA, and its inhibitor- PAI1). Endothelium-derived mediators (FGF2, TGFbeta, PDGF, NO) may stimulate (FGF2, TGFbeta, PDGF), or inhibit (NO) intimal proliferation and vascular smooth muscle hypertrophy and contribute to angiogenesis.

Endothelial dysfunction is defined as a functional damage to the endothelium characterized by increased vascular permeability and imbalance between different vasoactive, 
coagulation/anticoagulation and proliferative/antiproliferative factors resulting in the propensity to vasoconstriction, thrombotic diathesis and insufficient inhibition of subendothelial intimal proliferation. Endothelial dysfunction is also characterized by the insufficient response to vasodilatory stimuli (increased blood flow, or, e.g. acetylcholine), increased expression of adhesion molecules and subsequent infiltration of the vascular wall with monocytes/macrophages, increased tendency to the formation of platelet thrombi, impaired fibrinolysis and increased formation of radical oxygen species contributing to the damage of vascular wall and its increased permeability.

Generalized or localized endothelial dysfunction may also play an important role in the pathogenesis of early atherosclerosis, hypertension, diabetic complications and heart and renal failure. Endothelial dysfunction may be induced by many endothelium-damaging factors, e.g. aging, hypertension, hyperglycaemia, hyperlipidemia, hypoxia, smoking, infection and also cytotoxic treatment for cancer.

Formation of tumor metastases is at least partly dependent on the changed function of vascular endothelium which has some characteristics of the endothelial activation (increased expression of adhesion molecules, e.g. E-selectin, increased production of prothrombotic, e.g thromboxan A2, and antifibrinolytic, e.g. PAI-1, factors), or even endothelial dysfunction. Increased endothelial expression of some adhesion molecules (e.g. VCAM-1) is directly related to tumor angiogenesis.

The relation between the parameters of endothelial function (e.g. flow-mediated vasodilatation, production of $\mathrm{NO}$ and free radicals, local expression of von Willebrand factor) and the propensity to the formation of metastases have not been systematically studied in cancer patients. Tissue extravasion of tumor cells which is the prerequisite of the metastasis formation requires the interaction between tumor cells and endothelium which shares many similarities with the interaction of leukocytes with endothelium during inflammation and is also mediated by the adhesion molecules of both selectin (E- and P-selectins) and immunoglobulin (ICAM-1 and VCAM-1) superfamilies. Tumors cells must express, similarly as activated leukocytes, on their surface counterreceptors of these adhesion molecules (lectins and beta- 1 and beta- 2 integrins). Endothelial expression of adhesion cells is stimulated by circulating proinflammatory cytokines. Prevailing type of expressed adhesion molecule may contribute to the location of metastases, e.g. E-selection is known to be preferentially expressed in the hepatic vascular bed, whereas VCAM-2 is preferentially expressed in the lung circulation [1].

Undoubtedly, angiogenesis and formation of metastases are both very complex events and endothelial activation/ dysfunction (if present) may be only one of the several factors in the proposed pathogenetic cascade (except for the activation of many cytokines, growth factors, proteases, etc.). Generalised endothelial dysfunction cannot also explain the specific formation of metastases in different tissues. Supposedly, endothelial dysfunction could be necessary, but not sufficient condition for both angiogenesis and formation of metastasis.

Identification of early markers of endothelial activation/dysfunction could enhance early diagnosis of patients at high risk of generalisation (formation of metastases) and monitoring of these markers could possibly result in the early use of modified (intensified) therapeutic protocol.

In our study we concentrated on the high risk group of premenopausal patients younger than 40 years of age with relatively low risk of early atherosclerosis which could otherwise confound our results. In these patients we evaluated selected markers of endothelial activation and endothelial function using flow-mediated vasodilatation.

Based on our previous studies we hypothesised that at least some young patients with breast cancer may have not only signs of endothelial activation (increased serum levels of soluble adhesion molecules and increased expression of adhesion molecules by endothelial cells), but also the signs of generalized endothelial dysfunction estimated by the ultrasonographical methodswhich may precede the formation of distant metastases and assess if the endothelial activation/dysfunction is related to the outcome of the patients (progression, relapse, or even mortality).

\section{Materials and methods}

In this study we concentrated on the young women with breast cancer (younger than 40 years of age) treated in the Department of Oncology of our medical school and hospital. One of the reasons why we excluded older women was to minimize the influence of age and age-dependent atherosclerotic changes on endothelial activation and function. The study conformed with the principles outlined in the Declaration of Helsinki. It was approved by the medical ethical committee, and all patients gave their written informed consent before inclusion.

Altogether we examined 96 patients with breast cancer of the mean age of 38.5 years and 20 healthy age-matched controls. Based on the clinical stages of the disease 5 patients could have been classified as stage 0,29 as stage I, 38 as stage III, 17 as stage III and 7 as stage IV. 49 pts have positive estrogen receptors, in 47 patients estrogen receptors were negative. Median follow-up of the patients was 2.5 years. At the end of follow-up 76 patients were in remission, 4 patients were still on treatment, in 6 patients the disease was still active despite treatment, in 3 patients the disease relapsed and 9 patients died.

Parameters of endothelial activation (soluble VEGF, Pselectin, E-selectin, ICAM-1) were measured in the Institute of Clinical Biochemistry and Laboratory Diagnostics of our medical school and hospital using the commercialy available ELISA kits. Detailed laboratory coagulation examination (aPTT, fibrinogen, D-dimers, protein C, protein S, tPA, PAI-1, antitrombin III, plasminogen, a2-antiplasmin, von Willebrand factor) were also evaluated to get the insight into the coagulation status of the patients. 
Flow-mediated vasodilation (FMD) and peak blood flow (PBF) were measured using Doppler ultrasonography in the laboratory of the 3rd Department of Internal Medicine of our hospital. The methods used in our institution were described earlier [2]. Briefly, we used high resolution linear array probe and recorded brachial artery at rest and then after 4.5 minutes of forearm ischemia induced by a sphygmomanometer cuff. FMD was expressed as the percentage of postischemic brachial artery dilatation (in comparison to baseline), PBF was measured during the first 3 seconds after cuff deflation and expressed in $\mathrm{ml} / \mathrm{min}$.

Statistical evaluation and cluster analysis was performed by the Institute of Chemical Technology in Prague. Cluster analysis is an exploratory data analysis tool for identifying homogenous groups of objects called clusters [3]. Objects in a specific cluster share many characteristics, but are very dissimilar to objects not belonging to that cluster. Each object should be characterised by the value of (experimental) variables (features, parameters). By choosing a specific clustering procedure, we should determine how clusters are to be formed. There are many different clustering procedures and also many ways of classifying these. One of the clustering techniques is called hierarchical agglomerative clustering which was used in this work. In this method, clusters are consecutively formed from objects. Initially, this type of procedure starts with each object representing an individual cluster. These clusters are then sequentially merged according to their similarity (or dissimilarity). As a measure of association between objects (distance metrics) the Euclidean distance was used in this work. All calculations were done by program XLSTAT2 (www.wlstat.com, Addisoft, 2011).

\section{Results}

Patients with breast cancer had when compared to agematched healthy controls significantly higher serum levels of soluble E-selectin, P-selectin and ICAM-1. Serum levels of VEGF in patients with breast cancer did not differ from controls (Fig. 1).

Serum levels of P-selectin were significantly higher in patients with larger tumor mass (T3 and T4 vs. T1 and T2 -231.0 \pm 103.5 vs. $168.9 \pm 121 \mathrm{ng} / \mathrm{ml}, \mathrm{p}<0.05)$ and in breast cancer patients with nodal metastases (N1) compared to patients without nodal involvement $(\mathrm{N} 0-208.5 \pm 141.4$ vs. $152.8 \pm 91.1$ $\mathrm{ng} / \mathrm{ml}, \mathrm{p}<0.05)$. Soluble ICAM- 1 was the only parameter related to the outcome (patients with poor outcome - altogether 18 pts - during mean FU of 2.5 years either died, or had still active disease despite treatment, or relapsed within the period of FU, patients with good outcome - 76 patients in long term remission). Patients with poor outcome had at presentation vs. patients with good outcome significantly higher soluble ICAM $1(474.2 \pm 124.7$ vs. $345.5 \pm 122.3 \mathrm{ng} / \mathrm{ml}, \mathrm{p}<0.05)$.

There was no statistically significant difference in FMD and PBF between patients and controls. Peak blood flow (PBF) was $424.3 \pm 195,4$ vs. $414,9 \pm 117,2 \mathrm{ml} / \mathrm{min}$ and flow-mediated vasodilation (FMD) was $6.15 \pm 21.3 \%$ vs. $7.13 \pm 3.5 \%$ in patients with breast cancer and controls, respectively. There was also no significant difference in parameters of endothelial dysfunction between different subgroups of patients with breast cancer (e.g. patients with different stage, grade, size of tumor, presence or absence of estrogen receptors, presence of Her/Neu receptors) including treatment (e.g. patients on hormonal treatment and patients not treated by tamoxifen or aromatase inhibitors).

In the further multistep statistical cluster analysis (prof. Suchanek, Institute of Chemical Technology, Prague) our aim was to identify the subgroups of patients with breast cancer with similar degree of endothelial activation and supposedly also with similar propensity to the formation of metastases. For the analysis we took into the consideration the following clinical and laboratory parameters: (tumor size,

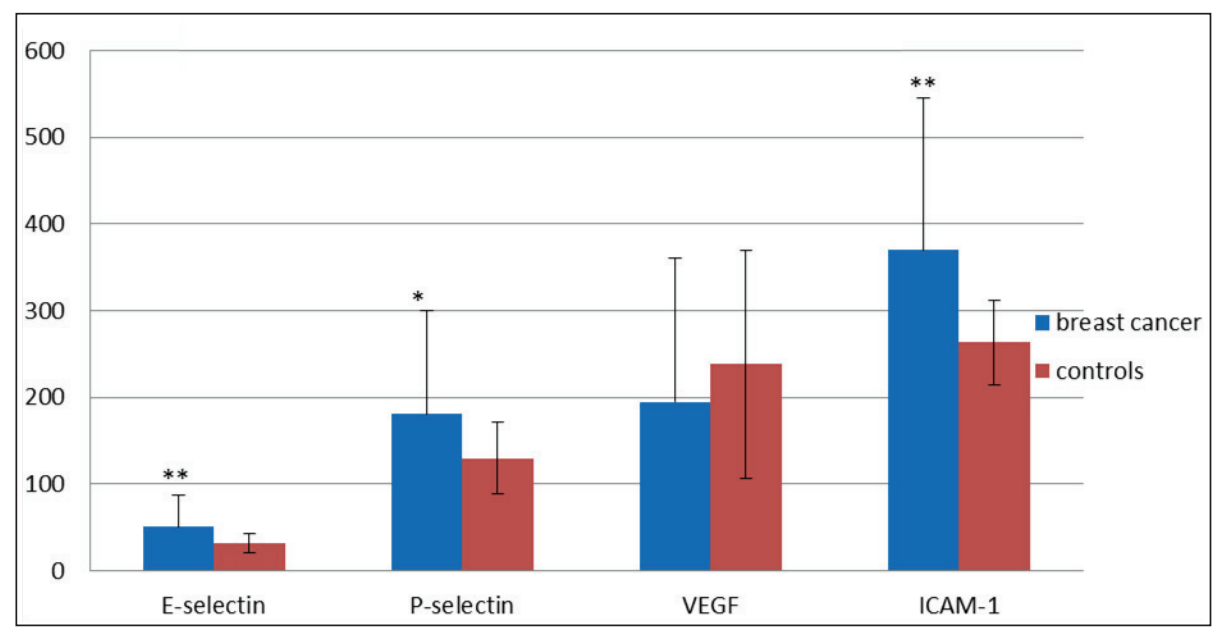

Figure 1. Serum levels of adhesion molecules and VEGF in patienst with breast cancer ${ }^{*} \mathrm{p}<0.05,{ }^{* *} \mathrm{p}<0.01$, E-selectin, P-selectin and ICAM-1 in $\mathrm{ng} / \mathrm{ml}$, VEGF in $\mathrm{pg} / \mathrm{ml}$ 
TNM classification, soluble E-selectin, P-selectin and ICAM-1, VEGF, aPTT, fibrinogen, AT III, D-dimers, a2-antiplazmin, protein $\mathrm{C}$, protein $\mathrm{S}, \mathrm{APC}$ rezistence, von Willebrand factor, tissue activator of plasminogen (tPA), plasminogen activator inhibitor (PAI-1) and plasminogen (Table 1).

Based originally only on serum levels of soluble adhesion molecules and VEGF we formed the data matrix with the aim to identify the putative subgroups which could be then validated with the inclusion of further clinical and laboratory data. Cluster analysis suggested that the patients could be optimally divided into 5 uneven subgroups characterized in Table 2 . The groups 1 and 2 had very similar levels of soluble E-selectin and soluble ICAM-1, but differed in serum levels of soluble P-selectin and VEGF. The largest group 2 was identified by low soluble P-selectin, very small groups 4 and 5 were characterized by very high serum levels of VEGF (group 4) or soluble ICAM-1 (group 5). Soluble E-selectin and soluble ICAM-1 tightly correlated.

Division of patients into 5 (different) subgroups was also suggested based on the pannel of coagulation parameters
(aPTT, fibrinogen, AT III, D-dimer, protein C, protein S, PAI-1 a plasminogen). The largest groups 1 and 2 were very similar in all measured coagulation parameters with the exception of D-dimers (very low D-dimers in group 2). Group 3 was characterized by high levels and group 4 by very high levels of D-dimer, group 4 and 5 had high levels of PAI-1. There was a tight correlation between protein S, PAI-1 and plasminogen. Linear discriminatory analysis demonstrated that the coagulation factors were less discriminative than soluble adhesion molecules and VEGF.

For the next analysis data matrix consisting of soluble Eselectin, P-selectin, ICAM-1, VEGF, D-dimer, protein C, PAI-1 and plasminogen (which were found in the previous analyses to be most discriminative) was evaluated. Cluster analysis once again confirmed that the division into 5 subgroups was optimal (table 3). Very small group 5 had extreme levels of practically all parameters, another small group 4 had very high VEGF and high ICAM-1. Remaining 3 relatively large groups differed in VEGF (high in group 1), soluble P-selectin (low in group 2) and E-selectin (higher in group 3). Linear

Table 1. Basic laboratory parameters in our young patients with breast cancer

\begin{tabular}{|c|c|c|c|c|c|c|c|c|c|}
\hline & $\mathbf{n}$ & mean & median & SD & RSD & 95\% quantil & $5 \%$ quantil & MAX & MIN \\
\hline sE-selectin & 96 & 50.9 & 45.85 & 36.3 & $71.2 \%$ & 105.0 & 12.4 & 252.5 & 6.0 \\
\hline sP-selectin & 96 & 180.6 & 168.1 & 119.9 & $66.4 \%$ & 386.4 & 15.8 & 534.7 & 0.2 \\
\hline VEGF & 96 & 194.4 & 152.6 & 166.3 & $85.5 \%$ & 443.6 & 34.8 & 1009.2 & 1.0 \\
\hline ICAM-1 & 96 & 369.8 & 331.5 & 175.7 & $47.5 \%$ & 531.3 & 220.1 & 1502.3 & 144.3 \\
\hline$A P T T$ & 95 & 32.1 & 30.8 & 7.2 & $22.4 \%$ & 39.5 & 25.8 & 78.7 & 25.0 \\
\hline$F b g$ & 95 & 3.2 & 3.2 & 0.8 & $24.1 \%$ & 4.5 & 2.2 & 5.6 & 1.1 \\
\hline$A T I I I$ & 95 & 103.5 & 103 & 11.7 & $11.3 \%$ & 122.3 & 83.0 & 128 & 82.0 \\
\hline D-Dimer & 95 & 271.9 & 172 & 620.8 & $228.3 \%$ & 432.7 & 43.9 & 5358 & 0.0 \\
\hline$P \_A 2 A P$ & 91 & 103.5 & 105 & 18.0 & $17.4 \%$ & 129.5 & 67.3 & 137 & 61.0 \\
\hline Protein C & 95 & 115.3 & 112 & 25.1 & $21.8 \%$ & 157,6 & 74,0 & 181 & 50.0 \\
\hline Protein $S$ & 95 & 81.2 & 81 & 23.8 & $29.3 \%$ & 125.7 & 42.7 & 146 & 18.0 \\
\hline APC rezistence & 95 & 2.9 & 2.89 & 0.6 & $20.5 \%$ & 3.8 & 2.0 & 4.4 & 1.5 \\
\hline$P \_P A 1 a$ & 95 & 30.7 & 22.5 & 27.6 & $89.8 \%$ & 75.8 & 5.2 & 158.1 & 2.8 \\
\hline P_TPAg & 88 & 4.1 & 3.15 & 3.3 & $80.8 \%$ & 9.5 & 1.8 & 21 & 0.8 \\
\hline$P \_F V W$ & 95 & 106.6 & 104 & 34.4 & $32.3 \%$ & 152.2 & 56.7 & 300 & 51.0 \\
\hline Plasminogen & 94 & 100.3 & 100.5 & 21.5 & $21.5 \%$ & 137.0 & 66.6 & 163 & 51 \\
\hline
\end{tabular}

E-selectin, P-selectin and ICAM-1 in ng/ml, VEGF in pg/ml, D-dimer in $\mu \mathrm{g} / \mathrm{l}$, fibrinogen ( $\mathrm{Fbg}$ ) in in $\mathrm{g} / \mathrm{l}$, other coagulation protein concentrations are given in $\mathrm{mg} / \mathrm{l}, \mathrm{APC}$ resistance is a ratio and aPTT is in seconds

Table 2. Soluble adhesion molecules and VEGF in individual subgroups of patients with breast cancer identified by cluster analysis

\begin{tabular}{lccccc}
\hline Group & Number & sE-selectin & sP-selectin & VEGF & ICAM-1 \\
\hline 1 & 27 & 44,6 & 220,6 & 299,1 & 342,8 \\
2 & 50 & 45,3 & 104,6 & 31,9 & 340,9 \\
3 & 12 & 52,2 & 377,5 & 94,5 & 295,6 \\
4 & 4 & 61,2 & 237,9 & 781,9 & 734,0 \\
5 & 3 & 183,6 & 221,8 & 78,1 & 1173,6 \\
\hline
\end{tabular}

E-selectin, P-selectin and ICAM-1 in ng/ml, VEGF in $\mathrm{pg} / \mathrm{ml}$

E-selectin, P-selectin and ICAM-1 in ng/ml, VEGF in pg/ml, D-dimer in $\mu \mathrm{g} / \mathrm{l}$, fibrinogen ( $\mathrm{Fbg}$ ) in in $\mathrm{g} / \mathrm{l}$, other coagulation protein concentrations are given in $\mathrm{mg} / \mathrm{l}$, APC resistance is a ratio and aPTT is in seconds 
discriminative analysis disclosed very good discriminative ability of the used parameters.

Furthermore, clinical (non-parametric factors - TNM stage of the tumor, size of the tumor, lymph node involvement, presence of estrogen/progesteron and Her-neu receptors, grade of the tumor and current state of the disease - remission, activity, relaps, exitus, on treatment) were taken into consideration in the further cluster analysis. Omitting groups represented only by 1-3 patients class 1 was characterized with positive estrogen receptors and only localized disease in remission at the end of FU. Outcome seemed to be worse and less predictable in patients in class 3 with negative estrogen receptors. Compared to class 1 patients in class 3 had higher soluble E-selectin and soluble ICAM-1 (higher endothelial activation) and lower VEGF and D-dimers. Some laboratory parameters thus clearly divided the patients into several well defined subgroups with different clinical outcome (Table 4).
Finally, data from ultrasound examination (50 pts and 20 controls) were also taken into consideration for cluster analysis and were assessed separately, as the ultrasound examination was performed only in a subgroup of patients. The following 6 parameters were taken into consideration (basal diameter, basal maximal velocity, basal mean velocity, maximal and mean velocity during peak blood flow and diameter during flow mediated dilatation.

Data matrix including all 6 parameters described above $(50$ patients and 20 controls) was subjected to cluster analysis (with the use of the method of agglomerative hierarchical clustering). Optimally 3 clusters were identified (table 5). Apparently, the mean values of ultrasound parameters of endothelial function were not different between patients with breast cancer and controls. However, high diameter and flow-mediated diameter and high basal and peak blood flow velocity was present only in healthy subjects. Patients with breast cancer seemed to be

Table 3. Subgroups of patients with breast cancer based on the cluster analysis of both adhesion molecules and VEGF and selected coagulation parameters

\begin{tabular}{lccccccccc}
\hline Class & Number & sE-selectin & sP-selectin & VEGF & ICAM-1 & D-Dimer & Protein C & P_PA1a & Plasminogen \\
\hline 1 & 27 & 41.0 & 234.7 & 282.2 & 319.7 & 204.4 & 119.0 & 28.1 & 102.6 \\
2 & 37 & 46.4 & 91.6 & 124.3 & 348.5 & 234.9 & 119.1 & 27.8 & 95.8 \\
3 & 22 & 56.9 & 250.5 & 102.6 & 345.2 & 84.1 & 106.5 & 35.9 & 103.9 \\
4 & 3 & 49.3 & 215.7 & 721.6 & 554.2 & 182.0 & 119.3 & 20.0 & 87.7 \\
5 & 2 & 139.5 & 388.0 & 517.9 & 987.8 & 4216.0 & 86.0 & 103.9 & 131.0 \\
\hline
\end{tabular}

E-selectin, P-selectin and ICAM-1 in $\mathrm{ng} / \mathrm{ml}$, VEGF in pg/ml, D-dimer in $\mu \mathrm{g} / \mathrm{l}$, fibrinogen (Fbg) in in $\mathrm{g} / \mathrm{l}$, other coagulation protein concentrations are given in $\mathrm{mg} / \mathrm{l}, \mathrm{APC}$ resistance is a ratio and aPTT is in seconds

Table 4. Association between clinical parameters and laboratory data based on the cluster analysis

\begin{tabular}{|c|c|c|c|c|c|c|c|c|c|}
\hline Class & 1 & 2 & 3 & 4 & 5 & 6 & 7 & 8 & 9 \\
\hline \multirow[t]{12}{*}{ no. } & 9 & 5 & 11 & 1 & 3 & 2 & 3 & 1 & 1 \\
\hline & group no.-1 & group no.-2 & group no.-3 & group no.-5 & ss-exitus & TNM-0 & TNM-3 & ER/PR-neg & ER/PR-pos \\
\hline & ss-remision & group no.-4 & ss-active & & TNM-4 & $\mathrm{T}-0$ & $\mathrm{~T}-4$ & & \\
\hline & TNM-1 & ER/PR-poz. & ss-reccurence & & LU-2 & & erb- 0,9 & & \\
\hline & $\mathrm{T}-1$ & erb-3 & ss-on treatment & & & & & & \\
\hline & LU-0 & Grade-2 & TNM-2 & & & & & & \\
\hline & ER/PR-poz & & $\mathrm{T}-2$ & & & & & & \\
\hline & erb-0 & & $\mathrm{T}-3$ & & & & & & \\
\hline & erb-1 & & LU-1 & & & & & & \\
\hline & Grade-1 & & ER/PR-neg & & & & & & \\
\hline & & & erb-2 & & & & & & \\
\hline & & & Grade-3 & & & & & & \\
\hline
\end{tabular}

Groups are defined based on biochemichal and coagulation parameters (table 2-5), TNM (staging of the cancer), T1-4, size of the primary tumor, LU - involvement of the nodes, ER - estrogen receptor, PR - progesteron receptor, erb - presence o Her/Neu receptors, ss - state at the end of follow-up: remission, active disease, death, recurrence, on treatment

Table 5. Mean values of individual parameters in identified clusters

\begin{tabular}{lcccccccc}
\hline Class & Number of patients & Number of controls & Diam_bas & Vmaxbas & Vmeanbas & Vmax_pbf & Vmean_pbf & Diam_fmd \\
\hline 1 & 8 & 0 & 3.66 & 90.79 & 27.48 & 185.00 & 111.25 \\
2 & 20 & 16 & 3.37 & 61.35 & 10.96 & 99.86 & 56.52 & 3.89 \\
3 & 22 & 3.40 & 81.11 & 17.21 & 148.05 & 79.21 & 3.64 \\
\hline
\end{tabular}


skewed to median or lower values with lower variability than healthy subjects.

Finally we tried to perform final cluster analysis with a data matrix containing soluble adhesion molecules (Eselectin, P-selectin, ICAM-1), VEGF, coagulation factors (aPTT, fibrinogen, AT III, D-dimer, protein C, protein S, APC resistance, tPA, PAI-1, vWF, plasminogen) and all clinical and ultrasound markers mentioned above (table 6). Apparently some parameters did not differ between different groups and do not contribute to the separation of the individual groups. From practical point we do not need to measure them.

Small groups 3 and 4 characterized by very high soluble ICAM-1 and high (group 3) or low (group 4) VEGF and Pselectin have a higher basal and flow mediated blood flow. Group 1 has compared to group 2 much higher E-selectin and P-selectin and slightly lower basal and flow mediated diameter of brachial artery. In the group 1 there was compared to group 2 more patients with more advanced stage of the disease and, rather surprisingly, more patients with positive estrogen receptors (table 7).

\section{Discussion}

In this study we found that patients with breast cancer have increased levels of endothelial activation markers (soluble Eselectin, P-selectin and ICAM-1, P-selectin) and these markers also seemed to be higher in patients with larger tumors, node involvement and seemed and poor outcome. On the contrary, surrogates of endothelial dysfunction (FMD, PBF) did not differ between patients and controls and also were not related to other characteristics of the cancer.
Increased serum levels of soluble E-selectin were demonstrated in patients with metastatic breast cancer, the highest levels being present in patients with liver metastases. Patients with breast cancer and high serum levels of soluble E-selectin were more prone to metastatic disease and had significantly shorter survival [4 - 8]. Soluble E-selectin was thus identified in patients with breast cancer as an independent negative prognostic factor.

Another adhesion molecule which may play an important role in the formation of metastases is P-selectin. Counterreceptor of P-selectin on tumor cells is CD24 (from the mucin superfamily). Administration of soluble CD24, or selective depletion of CD24 on the surface of tumor cells significantly decreased the metastatic potential of the breast cancer cells. Other (more common) counterreceptors of P- and E-selectin (sialyl Lewis(x) and sialyl Lewis (a)) are not expressed by normal epithelial cells of mammary gland, but they are expressed by breast cancer cells.

In our previous studies $[9,10]$ we demonstrated that patients with metastatic (and also non-metastatic) breast cancer have increased serum levels of soluble E- and P-selectin and ICAM-1. Serum levels of P-selectin correlated in these patients with increased serum levels of TNFa. Although the serum levels of soluble VCAM-1 were not increased in patients with breast cancer compared to controls and patients with metastatic breast cancer (stage IV) had higher serum levels of soluble VCAM-1 compared to patients with localized breast cancer (stage 0-1). Serum levels of soluble VCAM-1 and Pselectin significantly decreased in patients with breast cancer 3 months and 1 year after the initiation of the treatment. Serum levels of soluble ICAM-1 also decreased in these patients sig-

Table 6. Characteristics of the clusters of patients with breast cancer formed using all parameters

\begin{tabular}{lccccccc}
\hline Class & sE-selectin & sP-selectin & VEGF & ICAM-1 & APTT & Fbg & AT III \\
\hline $1(15)$ & $\mathbf{5 6 , 8 2 0}$ & $\mathbf{2 0 4 , 9 0 7}$ & 252,267 & $\mathbf{3 4 7 , 2 0 7}$ & 30,533 & 3,189 & 103,600 \\
$2(21)$ & $\mathbf{3 7 , 6 7 6}$ & $\mathbf{9 8 , 6 4 8}$ & 122,181 & $\mathbf{3 0 4 , 4 4 3}$ & 31,543 & 3,122 & 105,952 \\
$3(4)$ & $\mathbf{4 6 , 8 5 0}$ & $\mathbf{2 5 0 , 4 0 0}$ & 642,250 & $\mathbf{5 1 0 , 2 0 0}$ & 31,375 & 3,375 & 103,250 \\
$4(5)$ & $\mathbf{4 1 , 0 6 0}$ & $\mathbf{1 0 8 , 8 4 0}$ & 142,020 & $\mathbf{4 1 9 , 2 6 0}$ & 37,660 & 3,192 & 99,400 \\
\hline
\end{tabular}

\begin{tabular}{lcccccc}
\hline Class & Protein C & Protein S & APC rezistence & P_PA1a & P_FVW & Plasminogen \\
\hline $1(15)$ & 115,867 & 79,800 & 2,647 & 26,900 & 94,733 & 107,467 \\
$2(21)$ & 119,000 & 67,048 & 2,724 & 19,567 & 121,714 & 85,524 \\
$3(4)$ & 117,000 & 62,000 & 2,875 & 26,425 & 96,000 & 96,000 \\
$4(5)$ & 114,000 & 95,200 & 2,716 & 46,000 & 110,800 & 116,800 \\
\hline
\end{tabular}

\begin{tabular}{lccccccc}
\hline Class & Diam pr_baz & Vmax pr_baz & Vmean pr_baz & TAVM pr_baz & Vmax_pbf & Vmean_pbf & Diam pr_fmd \\
\hline $1(15)$ & $\mathbf{3 , 3 4 7}$ & 70,677 & 15,644 & $\mathbf{8 , 2 1 0}$ & 131,253 & 70,573 & $\mathbf{3 , 5 6 5}$ \\
$2(21)$ & $\mathbf{3 , 4 4 7}$ & 70,798 & 12,936 & $\mathbf{7 , 0 9 4}$ & 121,848 & 67,362 & $\mathbf{3 , 6 2 6}$ \\
$3(4)$ & $\mathbf{3 , 4 4 4}$ & 89,275 & 28,159 & $\mathbf{1 4 , 9 4 5}$ & 156,250 & 98,000 & $\mathbf{3 , 7 8 2}$ \\
$4(5)$ & $\mathbf{3 , 8 0 8}$ & 87,900 & 24,016 & $\mathbf{1 2 , 5 6 1}$ & 148,400 & 75,880 & $\mathbf{4 , 0 7 5}$ \\
\hline
\end{tabular}


Table 7. Different clinical parameters in different classes identified by cluster analysis

\begin{tabular}{|c|c|c|c|c|c|c|c|c|c|c|c|c|c|}
\hline class $1, n=15$ & & & & & & & & & & & & & \\
\hline Current state & & $T N M$ & & Tumor size & & Nodes $(L U)$ & & $E R / P R$ & & $e r b$ & & Grade & \\
\hline Remission & $86,7 \%$ & 0 & $6,7 \%$ & 0 & $6,7 \%$ & 0 & $53,3 \%$ & poz & $73,3 \%$ & 0 & $26,7 \%$ & 1 & $20,0 \%$ \\
\hline Death & $6,7 \%$ & 1 & $26,7 \%$ & 1 & $40,0 \%$ & 1 & $46,7 \%$ & neg & $26,7 \%$ & 1 & $40,0 \%$ & 2 & $40,0 \%$ \\
\hline Reccurrence & $6,7 \%$ & 2 & $40,0 \%$ & 2 & $26,7 \%$ & 2 & $0,0 \%$ & & & 2 & $13,3 \%$ & 3 & $40,0 \%$ \\
\hline \multirow[t]{2}{*}{ On treatment } & $0,0 \%$ & 3 & $20,0 \%$ & 3 & $13,3 \%$ & & & & & 3 & $20,0 \%$ & & \\
\hline & & 4 & $6,7 \%$ & 4 & $13,3 \%$ & & & & & & & & \\
\hline \multicolumn{14}{|l|}{ class $2, \mathrm{n}=21$} \\
\hline Current state & & $T N M$ & & Tumor size & & Nodes (LU) & & $E R / P R$ & & $e r b$ & & Grade & \\
\hline Remission & $90,5 \%$ & 0 & $0,0 \%$ & 0 & $0,0 \%$ & 0 & $52,4 \%$ & poz & $52,4 \%$ & 0 & $33,3 \%$ & 1 & $14,3 \%$ \\
\hline Death & $4,8 \%$ & 1 & $47,6 \%$ & 1 & $66,7 \%$ & 1 & $42,9 \%$ & neg & $47,6 \%$ & 1 & $14,3 \%$ & 2 & $47,6 \%$ \\
\hline Recurrence & $0,0 \%$ & 2 & $42,9 \%$ & 2 & $28,6 \%$ & 2 & $4,8 \%$ & & & 2 & $19,0 \%$ & 3 & $38,1 \%$ \\
\hline \multirow[t]{2}{*}{ On treatment } & $4,8 \%$ & 3 & $9,5 \%$ & 3 & $4,8 \%$ & & & & & 3 & $33,3 \%$ & & \\
\hline & & 4 & $0,0 \%$ & 4 & $0,0 \%$ & & & & & & & & \\
\hline \multicolumn{14}{|l|}{ class $3, \mathrm{n}=4$} \\
\hline Current state & & $T N M$ & & Tumor size & & Nodes (LU) & & $E R / P R$ & & $e r b$ & & Grade & \\
\hline Remission & $75,0 \%$ & 0 & $0,0 \%$ & 0 & $0,0 \%$ & 0 & $50,0 \%$ & poz & $75,0 \%$ & 0 & $50,0 \%$ & 1 & $0,0 \%$ \\
\hline Death & $25,0 \%$ & 1 & $0,0 \%$ & 1 & $75,0 \%$ & 1 & $50,0 \%$ & neg & $25,0 \%$ & 1 & $0,0 \%$ & 2 & $75,0 \%$ \\
\hline Recurrence & $0,0 \%$ & 2 & $50,0 \%$ & 2 & $0,0 \%$ & 2 & $0,0 \%$ & & & 2 & $25,0 \%$ & 3 & $25,0 \%$ \\
\hline \multirow[t]{2}{*}{ On treatment } & $0,0 \%$ & 3 & $25,0 \%$ & 3 & $0,0 \%$ & & & & & 3 & $25,0 \%$ & & \\
\hline & & 4 & $25,0 \%$ & 4 & $25,0 \%$ & & & & & & & & \\
\hline \multicolumn{14}{|l|}{$\operatorname{class} 4, n=5$} \\
\hline Current state & & $T N M$ & & Tumor size & & Nodes $(L U)$ & & $E R / P R$ & & $e r b$ & & Grade & \\
\hline Remission & $60,0 \%$ & 0 & $20,0 \%$ & 0 & $20,0 \%$ & 0 & $60,0 \%$ & poz & $80,0 \%$ & 0 & $20,0 \%$ & 1 & $20,0 \%$ \\
\hline Death & $20,0 \%$ & 1 & $40,0 \%$ & 1 & $40,0 \%$ & 1 & $40,0 \%$ & neg & $20,0 \%$ & 1 & $0,0 \%$ & 2 & $60,0 \%$ \\
\hline Recurence & $0,0 \%$ & 2 & $0,0 \%$ & 2 & $20,0 \%$ & 2 & $0,0 \%$ & & & 2 & $20,0 \%$ & 3 & $20,0 \%$ \\
\hline \multirow[t]{2}{*}{ On treatment } & $20,0 \%$ & 3 & $20,0 \%$ & 3 & $0,0 \%$ & & & & & 3 & $60,0 \%$ & & \\
\hline & & 4 & $20,0 \%$ & 4 & $20,0 \%$ & & & & & & & & \\
\hline
\end{tabular}

nificantly, but still remained significantly increased compared to healthy subjects.

Intact endothelium is the prerequisite for its normal response to vasodilatory stimuli. Assessment of flow mediated vasodilation is a practical, noninvasive method to evaluate endothelial function. The method must, however, be done reproducibly by experienced observers to avoid unnecessary high intraobserver and interobserver variability $[2,11]$.

There is a very limited experience with the evaluation of the flow mediated vasodilation in patients with breast cancer. It has been studied only in small groups of mostly elderly postmenopausal patients, often without appropriate healthy controls and namely in relation to the treatment with estrogen receptor modulators [12]. In older women endothelial dysfunction may be the sign of the initial stage of cardiovascular disease and may not be related to the presence of cancer. Moreover, different chemotherapeutic agents, used namely in women with advanced breast cancer may have direct toxic effect on the endothelial cells and can cause in vitor either their necrosis, or apoptosis [13], including anthracyclines, gemcitabine, vinca alcaloids [14]. Impaired vascular reactivity may be also related to the activation of proinflammatory factors, e.g. TNFa [15]. On the other hand, administration of the granulocyte colony stimulating factor has been recently shown to be associated with the improvement in endothelial function [16].

In elderly (mean age 61 years) patients with early breast cancer $(90 \%$ of them with stage I and II) tamoxifen, but not letrozol, decreased serum cholesterol and increased flow mediated vasodilation, possibly due to its estrogen-like effects [12]. No effect of one-month treatment with tamoxifen on FMD was demonstrated in another small study (only 21 patients of median age 61 years - [17]). In another very small study (14 overweight pts of the median age of 63 years) FMD did not differ between patients with breast cancer and age-matched controls [18]. FMD increased after 6 month of tamoxifen treatment in patients with breast cancer and remained unchanged in untreated controls. In a very small group $(n=5)$ of postmenopausal women with breast cancer (mean age 65 years) chemotherapeutic drug, paclitaxel, with previously demonstrated proinflammatory and antiangiogenic properties was shown to reduce flow mediated vasodilation [19].

None of these studies was performed in young patients (without putative impact of cardiovascular disease on endothe- 
lial dysfunction, and none of them specifically looked at the difference between patients and control subjects.

In this paper we tried to correlate the markers of endothelial activation (namely soluble adhesion molecules) with the direct assesment of endothelial function using flow mediated vasodilation in young women with breast cancer to avoid the potential confounding factors of early (or even advanced atherosclerosis) and its risk factors. We tried to use these parameters to define the specific subgroups of patients with breast cancer with different extent of the disease on presentation and different outcome using cluster analysis.

We were able to confirm the higher soluble E-selectin, P-selectin and ICAM-1 levels in patients with breast cancer, but there was no significant difference in flow mediated vasodilation between young women with breast cancer and their age-matched controls (although there was a tendency to lower peak blood flow velocity in breast cancer patients). In cluster analysis, group of patients with high E-selectin, high ICAM1 (higher endothelial activation) and low VEGF (endothelial damage?) had more frequently negative estrogen receptors and had worse outcome compared to the group of patients with lower E-selectin, lower ICAM-1 and mostly positive estrogen receptors.

If confirmed in larger groups of patients these data suggest that monitoring of some parameters of endothelial activation could help to identify the patients in higher risk of progression and poor outcome.

Acknowledgments: The study was supported by the research projects PRVOUK - P25/LF1/3 and RVO-VFN64165.

\section{References}

[1] FOX SB, TURNER GDH, GATTER KC, HARRIS AL The increased expression of adhesion molecules ICAM-3, E- and P-selectins on breast cancer endothelium. J Pathol 1995; 177: 369-376. http: //dx.doi.org/10.1002/path.1711770407

[2] MALIK J, WICHTERLE D, HAAS T, MELENOVSKY V., SIMEK J et al. Repeatability of noninvasive surrogates of endothelial function. Am J Cardiol 2004, 94: 693-696. http: //dx.doi.org/10.1016/j.amjcard.2004.05.049

[3] VANDEGINSTE BGM Handbook of Chemometrics and Qualimetrics, Part B. Elsevier 1998, ISBN 0-444-82853-2.

[4] BANKS RE, GEARING AJ, HEMINGWAY IK, NORFOLK DR, TURNER GDH et al. Circulating intercellular adhesion molecule-1 (ICAM-1), E-selectin and vascular cell adhesion molecule-1 (VCAM-1) in human malignancies. Br J Cancer 1993; 68: 122-124. http: //dx.doi.org/10.1038/bjc.1993.298

[5] MATSUURA N, NARITA T, MITSUOKA C, KIMURA N, KANNAGI $\mathrm{R}$ et al. Increased concentrations of soluble Eselectin in the sera of breast cancer patients. Anticancer Res 1997; 17: 1367-1372.

[6] HEBBAR M, REVILLION F, LOUCHEZ MM, VILAIN MO, FOURNIER C, et al. The relationship between concentrations of circulating soluble E selectin and clinical, pathological, and biological features in patients with breast cancer. Clin Cancer Res 1998; 4: 373-380.

[7] HEBBAR M, REVILLION F, LOUCHEZ MM, FOURNIER C, BONNETERRE J et al. Prognostic value of circulating soluble E-selectin concentrations in node-negative breast cancer patients. Clin Cancer Res 1999; 5: 1427-1433.

[8] HEBBAR M, PEYRAT JP Significance of soluble endothelial molecule E-selectin in patients with breast cancer. Int J Biol Markers 2000; 15: 15-21.

[9] TESAROVA P, KVASNICKA J, UMLAUFOVA A, HOMOLKOVA $H$, JIRSA $M$ et al. Soluble TNF and IL-2 receptors in patients with breast cancer. Med Sci Monit 2000; 6: 661667.

[10] TESAROVA P, KVASNICKA J, UMLAUFOVA A, HOMOLKOVA J, TESAR V Solubilní adhezní molekuly u pacientek s karcinomem mammy. Čas Lék čes 2003; 142: 292-299.

[11] KASPRZAK JD, KLOSINSKA M, DRODZ J Clinical aspects of assessment of endothelial function. Pharmacol Rep 2006; 58: Suppl. 33-40.

[12] OLIVEIRA NASTRIC, MARTINS WP, FERRIANI RA, FILHO FM, DOS REIS FJ et al. Sonographic evaluation of endothelial function in letrozol and tamoxifen users. Maturitas 2008; 61: 340-344. http: //dx.doi.org/10.1016/j.maturitas.2008.09.019

[13] KAUSHAL V, KAUSHAL GP, MEHTA P Differential toxicity of anthracyclines on cultured endothelial cells. Endothelium 2004; 11: 253-258. http: //dx.doi.org/10.1080/ 10623320490904124

[14] LOPEZ-MIRANDA V, HERRADON E, GONZALEZ C, MARTIN MI et al. Vascular toxicity of chemotherapeutic agents. Curr Vasc Pharmacol 2010; 8: 692-700. http: //dx.doi. org/10.2174/157016110792007012

[15] KARZAN A. Tumor necrosis factor and endothelial cell death. Trends Cardiovasc Med 1998; 8: 19-24. http: //dx.doi. org/10.1016/S1050-1738(97)00126-6

[16] IKONOMIDIS I, PAPADIMITRIOU C, VAMVAKOU G, KATISCHTI P, VENETSANOU $\mathrm{K}$ et al. Treatment with granulocyte colony stimulating factor is associated with improvement in endothelial function. Growth Factors 2008; 26: 117-124. http: //dx.doi.org/10.1080/08977190802090614

[17] ONG PJ, LINARDOU H, GRAHAM HA, SAVAGE P, HAYWARD CS et al. Tamoxifen is not detrimental to endothelial function in postmenopausal women with breast cancer. Am Heart J 2001; 142: E6.

[18] STAMATELOPOULOS KS, LEKAKIS JP, POULALAKI NA, PAPAMICHAEL CM, VENETSANOU K et al. Tamoxifen improves endothelial function and reduces carotid intima-media thickness in postmenopausal women. Am Heart J 2004; 147: 1093-1099. http: //dx.doi.org/10.1016/j.ahj.2003.12.029

[19] VASSILAKOPOULU M, MOUNNTZIOS G, PAPAMICHAEL C, PROTEGEROU AD, AZNAOURIDIS K et al. Paclitaxel chemotherapy and vascular toxicity as assessed by flow-mediated and nitrate-mediated vasodilatation. Vascular Pharmacology 2010; 53: 115-121. http: //dx.doi.org/10.1016/ j.vph.2010.05.002 